\title{
Subtemporal transtentorial approach for recurrent trigeminal neuralgia after microvascular decompression via the lateral suboccipital approach: case report
}

\author{
Toshihiro Ogiwara, MD,,2 Tetsuya Goto, MD, ${ }^{1}$ Yoshikazu Kusano, MD, ${ }^{2}$ Masafumi Kuroiwa, MD, \\ Takafumi Kiuchi, MD, ${ }^{1}$ Kunihiko Kodama, MD, ${ }^{2}$ Toshiki Takemae, MD, ${ }^{2}$ and Kazuhiro Hongo, MD ${ }^{1}$ \\ 1Department of Neurosurgery, Shinshu University School of Medicine, Matsumoto; and 2Department of Neurosurgery, Nagano \\ Municipal Hospital, Nagano, Japan
}

\begin{abstract}
Microvascular decompression (MVD) via lateral suboccipital craniotomy is the standard surgical intervention for trigeminal neuralgia (TN). For recurrent TN, difficulties are sometimes encountered when performing reoperation via the same approach because of adhesions and prosthetic materials used in the previous surgery. In the present case report the authors describe the efficacy of the subtemporal transtentorial approach for use in recurrent TN after MVD via the lateral suboccipital approach. An 86-year-old woman, in whom an MVD via a lateral suboccipital craniotomy had previously been performed for TN, underwent surgery for recurrent TN via the subtemporal transtentorial approach, which provided excellent visualization of the neurovascular relationships and the trigeminal nerve without adhesions due to the previous surgery. Her TN disappeared after the MVD. The present approach is ideal for visualizing the trigeminal root entry zone, and the neurovascular complex can be easily dissected using a new surgical trajectory. This approach could be another surgical option for reoperation when the previous MVD had been performed via the suboccipital approach.
\end{abstract}

http://thejns.org/doi/abs/10.3171/2014.10.JNS132643

KEY WORDS subtemporal transtentorial approach; microvascular decompression; recurrent trigeminal neuralgia; peripheral nerve

$\mathrm{S}$ URGICAL intervention is indicated for trigeminal neuralgia (TN) when medication fails. Microvascular decompression (MVD) via the lateral suboccipital approach is currently the most feasible approach for surgical treatment in cases of TN. , $^{1,4,17}$ Postoperative pain relief is obtained in up to $94 \%$ of cases after MVD. . $^{6,10,11}$ Although MVD has the best reported success rate for TN, symptoms can recur in patients in whom treatment was initially successful ${ }^{13}$ with a reported rate of recurrence of $6 \%-30 \% .{ }^{6,10,11}$ Some patients with residual or recurrent pain wish to undergo further treatment. Even methods for radiation therapy have been developed, and it is not rare to encounter patients who require reoperation. ${ }^{5,15}$ However, the surgical complication rate increases and the surgical success rate decreases at reoperation. ${ }^{12}$ Surgical difficulties, in the form of adhesions and prosthetic materials, are sometimes encountered when performing reoperation via the same route that was initially used. In addition to the lateral suboccipital approach, the subtemporal transtentorial approach can be used to reach the trigeminal nerve. In the present report the authors discuss the efficacy of this subtemporal transtentorial approach in a case involving recurrent $\mathrm{TN}$ in a patient who had previously undergone MVD via the lateral suboccipital approach.

\section{Case Report}

An 86-year-old woman underwent MVD via the lateral suboccipital approach for treatment of TN in the right V2 distribution when she was 70 years old. The trigeminal nerve was compressed by the right superior cerebellar artery (SCA) and petrosal vein (PV). Interposition of the offending vessels from the trigeminal nerve was achieved using a prosthesis. Her pain was completely relieved immediately after the surgery. However, pain recurred 14 years later at the age of 84 years. The pain was in the right V2-3 territory and gradually worsened despite medication. She was eventually referred to our service and hospitalized immediately because of poor oral intake, emaciation, and decline in quality of daily life due to severe pain. Preoperative MRI revealed the complex of vessels and previous prosthesis compressing the root entry zone (REZ) of the trigeminal nerve (Fig. 1). The patient wished to undergo direct surgery to relieve her severe pain, although radio-

ABBREVIATIONS MVD = microvascular decompression; $\mathrm{PV}=$ petrosal vein; $\mathrm{REZ}=$ root entry zone; $\mathrm{SCA}=$ superior cerebellar artery; $\mathrm{TN}=$ trigeminal neuralgia. SUBMITTED December 15, 2013. ACCEPTED October 14, 2014

INCLUDE WHEN CITING Published online November 28, 2014; DOI: 10.3171/2014.10.JNS132643.

DISCLOSURE The authors report no conflict of interest concerning the materials or methods used in this study or the findings specified in this paper. 

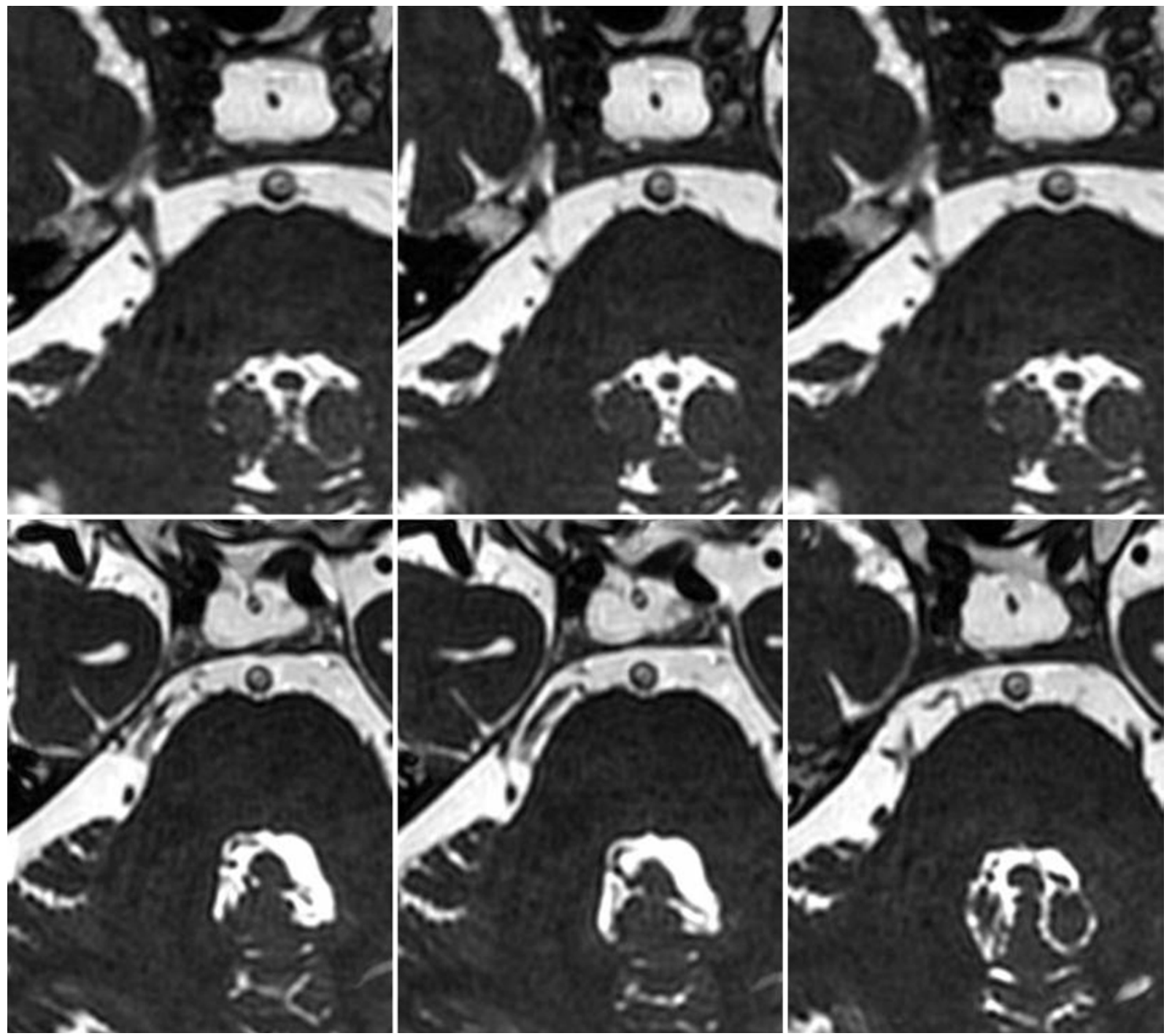

FIG. 1. Preoperative MR images revealing that the vessel complex and the previously placed prosthesis were compressing the REZ of the trigeminal nerve.

therapy was recommended because of her advanced age. The second MVD via the right subtemporal transtentorial approach was selected.

Under general anesthesia, the patient was placed in the supine-lateral position. Her head was rotated to the left side with the vertex up. A routine subtemporal intradural approach was followed and lumbar drainage was undertaken. The temporal lobe was gently retracted and cerebrospinal fluid was intermittently removed via a lumbar catheter. The tentorial opening was extended from the free edge to the level of the offending vessels on the REZ, taking care to avoid damaging the trochlear nerve. The previously placed prosthesis, SCA, PV, and REZ of the trigeminal nerve were identified. The old prosthesis, SCA, and PV compressed the REZ of the fifth cranial nerve. The old prosthesis was carefully removed, and the offending vessels were separated from the REZ (Fig. 2). The axis of the fifth cranial nerve was aligned from the REZ close to Meckel's cave. Postoperatively, the patient's pain resolved completely. Radiological examination showed no contu- sion or hemorrhage in the approach route. The patient experienced no seizures or neurological deficits, and her activities of daily living recovered fully. Two years after surgery, she is free of TN.

\section{Discussion}

In 1959, Gardner and Miklos ${ }^{2}$ reported their surgical experience in treating TN with MVD using the epidural approach via the middle fossa. In 1966, Jannetta and Rand ${ }^{9}$ performed MVD for TN via the subtemporal transtentorial approach in 9 patients. Successful mobilization of the vessel from the nerve was achieved in only 1 patient, and rhizotomy was performed in the other 8 patients. Jannetta ${ }^{8}$ reported that the transtentorial approach is ideal for visualizing the trigeminal REZ but is not useful for mobilizing the blood vessel away from the nerve. He felt it important to state that he had stopped using this approach because he had had 2 patients who suffered postoperative seizures, one of them with a temporal lobe hematoma. Two other 

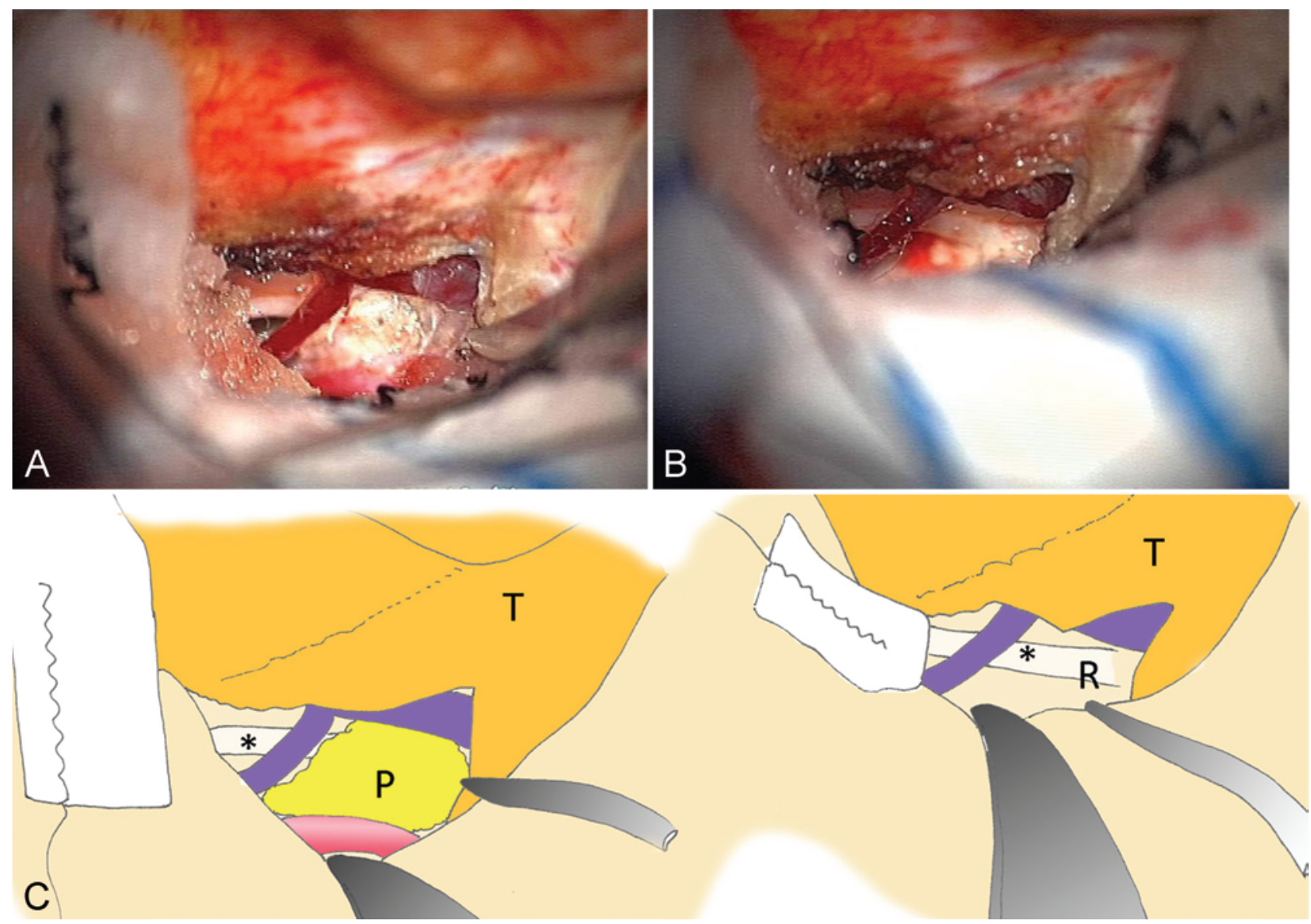

FIG. 2. A and B: Intraoperative photographs showing the view of subtemporal transtentorial approach. The previous prosthesis adhered to vessels and compressed the REZ of the trigeminal nerve (A). The prosthesis was successfully removed and the REZ of the trigeminal nerve was freed from the offending vessels (B). Lower: Artist's drawing of the steps shown in the intraoperative photographs. $\mathrm{P}=$ prosthesis; $\mathrm{R}=\mathrm{REZ} ; \mathrm{T}=$ tentorium; ${ }^{*}=$ fifth cranial nerve. Copyright Tetsuya Goto (artist). Published with permission. Figure is available in color online only.

surgeons have told us that they, too, have had difficulty with the complexity of temporal lobe elevation. The subtemporal transtentorial approach for TN was discontinued because of its unfavorable surgical results and its laboriousness. Later, MVD for TN was commonly undertaken via lateral suboccipital craniotomy. ${ }^{7}$ The lateral suboccipital approach is familiar to neurosurgeons and is the first choice in TN surgery. However, in the lateral suboccipital approach, serious complications may occur because many critical structures, such as blood vessels, cranial nerves, and the brainstem, must be dissected. Although the subtemporal transtentorial approach is not commonly used for MVD, it can be applied more safely than before owing to the development of surgical instruments and evolution in microsurgical techniques. In particular, the subtemporal approach can be a good option for patients whose brain is atrophic, whose lesion is on the nondominant side, and whose vein of Labbé is not developed.

The subtemporal transtentorial approach has commonly been used in surgery for basilar artery trunk aneurysms, superficial temporal artery-SCA bypass, brainstem lesions, etc., and the fifth nerve was described in the literature from the view of the transtentorial approach (Fig. 3). ${ }^{14,16}$ The subtemporal transtentorial approach can provide a wide and shallow operative field for the trigeminal nerve. Especially in surgery for recurrent or residual TN previously treated with the lateral suboccipital approach, there are several advantages to the subtemporal transtentorial approach over the lateral suboccipital approach. There is no difficulty in dissection because there are no adhesions in the approach route caused by the previous surgery. The trigeminal nerve is visible directly from the REZ, close to the Meckel's cave. The site compressed by the offending vessel is visible from different angles. In the present case, the previously inserted prosthesis was easily removed, and trigeminal neuralgia resolved postoperatively.

It is suggested that the subtemporal transtentorial approach is a useful surgical approach for MVD in surgically difficult cases, such as those involving recurrent TN. Here, we have reported a case of recurrent TN in which MVD was performed via the subtemporal transtentorial approach, which provided excellent and complete visualization of the neurovascular structures and the trigeminal nerve.

Use of the subtemporal transtentorial approach for recurrent TN appears to be efficacious. It enables surgeons to observe the trigeminal nerve in a straight line, from the REZ to Meckel's cave, without interference of the trigemi- 

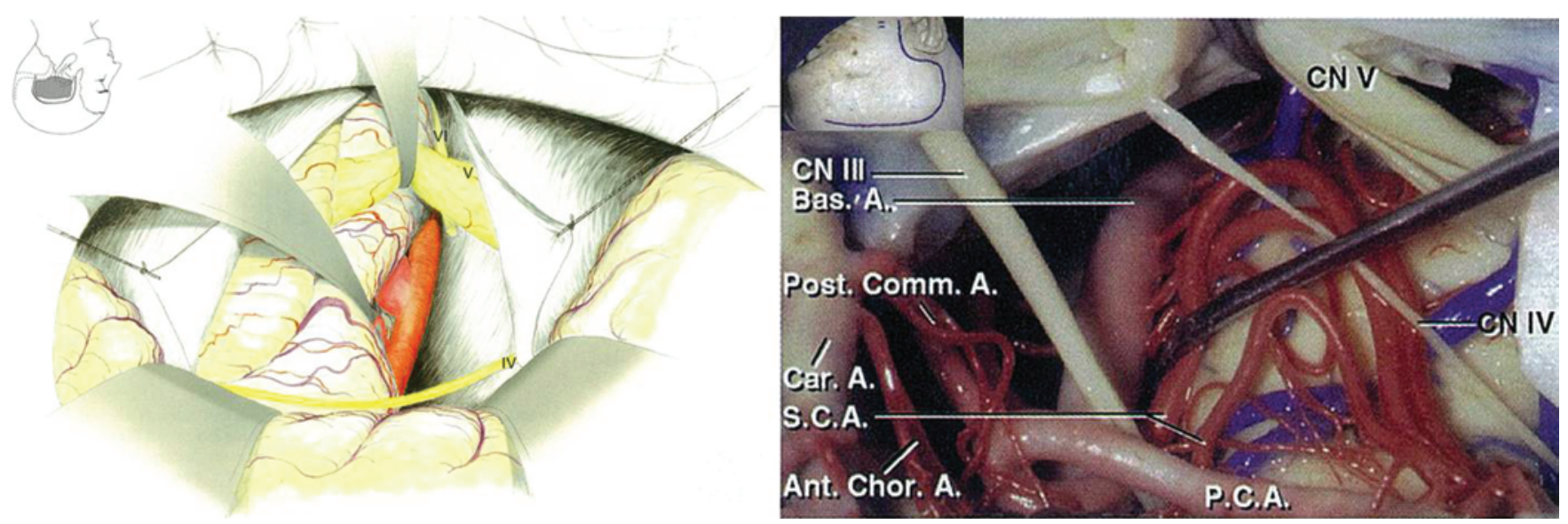

FIG. 3. Left: Operative view of the left subtemporal transtentorial approach for basilar trunk aneurysm. Visualization of the trigeminal nerve is excellent. With kind permission from Springer Science+Business Media: Microneurosurgical Atlas, "Aneurysm," 1985, pp 110-111, K Sugita, Case 44. Right: Subtemporal exposure of the right middle, infratemporal, and posterior fossa. The medial tentorial edge has been divided behind the petrous ridge to expose the trigeminal nerve. AL Rhoton Jr: Rhoton's Cranial Anatomy and Surgical Approaches. Philadelphia: Lippincott, Williams, \& Wilkins, 2013. With permission from The Congress of Neurological Surgeons. (Fig. 8.13.F). Figure is available in color online only.

nal tubercle and the anterolateral side of the REZ, compared with the suboccipital approach. The subtemporal approach may be suitable not only for recurrent TN, but also for other cases made difficult by the anatomical relationship of the offending vessel and the fifth cranial nerve. If the site compressed by the offending vessel is distal to the REZ or the anterolateral side, the subtemporal transtemporal approach may be more suitable for TN, even in nonrecurrent cases. A suitable surgical approach for TN can be selected by evaluating the preoperative neuroimaging findings.

\section{References}

1. Cohen-Gadol AA: Microvascular decompression surgery for trigeminal neuralgia and hemifacial spasm: naunces of the technique based on experiences with 100 patients and review of the literature. Clin Neurol Neurosurg 113:844-853, 2011

2. Gardner WJ, Miklos MV: Response of trigeminal neuralgia to decompression of sensory root; discussion of cause of trigeminal neuralgia. J Am Med Assoc 170:1773-1776, 1959

3. Gressot LV, Hassaneen W, Fox BD, Mitchell BD, Tatsui CE, Ehni BL, et al: Surgical treatment for combined hemifacial spasm and atypical trigeminal neuralgia caused by a tortuous basilar artery. Case report and review of the literature. J Neurosurg Sci 56:151-154, 2012

4. Hitotsumatsu T, Matsushima T, Inoue T: Microvascular decompression for treatment of trigeminal neuralgia, hemifacial spasm, and glossopharyngeal neuralgia: three surgical approach variations: technical note. Neurosurgery 53:14361443,2003

5. Huang CF, Chiou SY, Wu MF, Tu HT, Liu WS: Gamma Knife surgery for recurrent or residual trigeminal neuralgia after a failed initial procedure. Clinical article. J Neurosurg 113 Suppl:172-177, 2010

6. Inoue H, Kondo A, Shimano H, Yasuda S: Recurrent trigeminal neuralgia at 20 years after surgery: case report. Neurol Med Chir (Tokyo) 53:37-39, 2013

7. Jannetta PJ: Complications from microsurgical treatment of tic douloureux. J Neurosurg 40:675, 1974 (Letter)

8. Jannetta PJ: Treatment of trigeminal neuralgia by suboccipital and transtentorial cranial operations. Clin Neurosurg 24:538-549, 1977
9. Jannetta PJ, Rand RW: Transtentorial retrogasserian rhizotomy in trigeminal neuralgia by microneurosurgical technique. Bull Los Angeles Neurol Soc 31:93-99, 1966

10. Kondo A: Follow-up results of microvascular decompression in trigeminal neuralgia and hemifacial spasm. Neurosurgery 40:46-52, 1997

11. Kondo A, Date I, Endo S, Fujii K, Fujii Y, Fujimaki T, et al: A proposal for standardized analysis of the results of microvascular decompression for trigeminal neuralgia and hemifacial spasm. Acta Neurochir (Wien) 154:773-778, 2012

12. Onoda K, Satoh T, Kambara H, Omi M, Nabeshima M: [Reoperation for the recurrent trigeminal neuralgia-preoperative assessment with 3D MR cisternogram/angiogram fusion imaging.] No Shinkei Geka Sokuhou 19:1065-1071, 2009 (Jpn)

13. Payner TD, Tew JM Jr: Recurrence of hemifacial spasm after microvascular decompression. Neurosurgery 38:686-691, 1996

14. Rhoton AL Jr: The temporal bone and transtemporal approaches. Neurosurgery 53 (Part 3):643-697, 2003

15. Sanchez-Mejia RO, Limbo M, Cheng JS, Camara J, Ward MM, Barbaro NM: Recurrent or refractory trigeminal neuralgia after microvascular decompression, radiofrequency ablation, or radiosurgery. Neurosurg Focus 18(5):E12, 2005

16. Sugita K, Kobayashi S: Microneurosurgical Atlas Bibliography. Berlin: Springer-Verlag, 1985, pp 110-111

17. Tatli M, Satici O, Kanpolat Y, Sindou M: Various surgical modalities for trigeminal neuralgia: literature study of respective long-term outcomes. Acta Neurochir (Wien) 150:243-255, 2008

\section{Author Contributions}

Conception and design: Goto, Ogiwara, Kuroiwa, Kiuchi, Kodama, Takemae. Acquisition of data: Ogiwara. Drafting the article: Ogiwara. Critically revising the article: all authors. Reviewed submitted version of manuscript: all authors. Approved the final version of the manuscript on behalf of all authors: Goto. Study supervision: Takemae, Hongo.

\section{Correspondence}

Tetsuya Goto, Department of Neurosurgery, Shinshu University School of Medicine, 3-1-1 Asahi, Matsumoto 390-8621, Japan. email: tegotou@shinshu-u.ac.jp. 